Case Report

\title{
Bacteremia Caused by Kocuria kristinae from Egypt: Are There More? A Case Report and Review of the Literature
}

\author{
Reem M. Hassan, ${ }^{1}$ Dina M. Bassiouny, ${ }^{1}$ and Yomna Matar $^{2}$ \\ ${ }^{1}$ Department of Clinical and Chemical Pathology, Faculty of Medicine, Cairo University, Cairo, Egypt \\ ${ }^{2}$ Department of Psychiatry, Faculty of Medicine, Cairo University, Cairo, Egypt
}

Correspondence should be addressed to Reem M. Hassan; reem.mostafa@kasralainy.edu.eg

Received 1 July 2016; Accepted 12 October 2016

Academic Editor: Pau Montesinos Fernández

Copyright (c) 2016 Reem M. Hassan et al. This is an open access article distributed under the Creative Commons Attribution License, which permits unrestricted use, distribution, and reproduction in any medium, provided the original work is properly cited.

Kocuria kristinae is opportunistic Gram-positive cocci from the family Micrococcaceae. It is usually considered part of the normal flora that rarely is isolated from clinical specimens. Here, we report a case of Kocuria kristinae bacteremia; to the best of our knowledge, this is the first report from Egypt.

\section{Introduction}

Kocuria are Gram-positive, coccoid actinobacteria that occur in tetrads belonging to the family Micrococcaceae, suborder Micrococcineae, order Actinomycetales [1]. They are widely distributed in nature and can also be found frequently as normal skin and oral cavity flora in humans and other mammals. The genus contains 18 species, only five of which are known to be opportunistic pathogens [2].

Few reports on Kocuria spp. clinical infections exist in the literature. They were reported to cause catheter-related bacteremia in immunocompromised patients and those with chronic illness, peritonitis, cholecystitis, and urinary tract infection in patients with indwelling urinary catheters [3-6].

The underestimated prevalence of this organism is due to its misidentification as coagulase-negative staph and absence of guidelines for its clinical evaluation as a pathogen as it can be a common source of contamination in clinical specimens $[7,8]$.

\section{Case Report}

A 70-year-old female patient diagnosed with bipolar disease was admitted to the psychiatry department in Cairo University Hospital (Kasr Al-Ainy). The patient develops manic/ depressive episodes every now and then and was in an episode of mania for which she received treatment for 2 weeks, regular antipsychotic regimen (risperidone, depakine, and quetiapine). A peripheral cannula was introduced for intravascular fluids. The patient was not controlled with regular treatment and received aqueous intramuscular injection of clopixol $200 \mathrm{mg}$, after which she developed bilateral lower limb weakness, disturbed conscious level a day later, and fever. Weakness progressed to hypotonia and external rotation, with no rigidity and equivocal plantar reflex. Manifestations in the right limb were severer than in the left one, as there were redness, warmness, and edema of calf muscles but no pain or tenderness. The patient received clexane $40 \mathrm{mg}$ prophylaxis. Examination of the patient revealed temperature $38.3^{\circ} \mathrm{C}$, blood pressure $130 / 80 \mathrm{mmHg}$, pulse $110 / \mathrm{min}$, and respiratory rate $20 / \mathrm{min}$.

Radiological investigations were done in the form of CT brain, MRI brain, and echocardiogram, which were normal, and venous Duplex on lower limbs that showed recent adherent deep venous thrombosis (DVT) in right peroneal and soleal areas for which she received a higher dose of clexane (60 mg every 12 hours).

Laboratory investigations were done and results were as follows: CPK was $3200 \mathrm{U} / \mathrm{L}$ which gradually decreased later on, AST was $94 \mathrm{U} / \mathrm{L}$, platelets were 87000 cells $/ \mathrm{cmm}$, Na was $134 \mathrm{mg} / \mathrm{dL}$, $\mathrm{K}$ was $3.5 \mathrm{mg} / \mathrm{dL}$, Ca++ was $0.61 \mathrm{mg} / \mathrm{dL}, \mathrm{Hb}$ was $10 \mathrm{mg} / \mathrm{dL}$, WBCs were 3600 cells/cmm, CRP was positive, PT and PTT were normal, and urine culture grew E. coli (ESBL). 


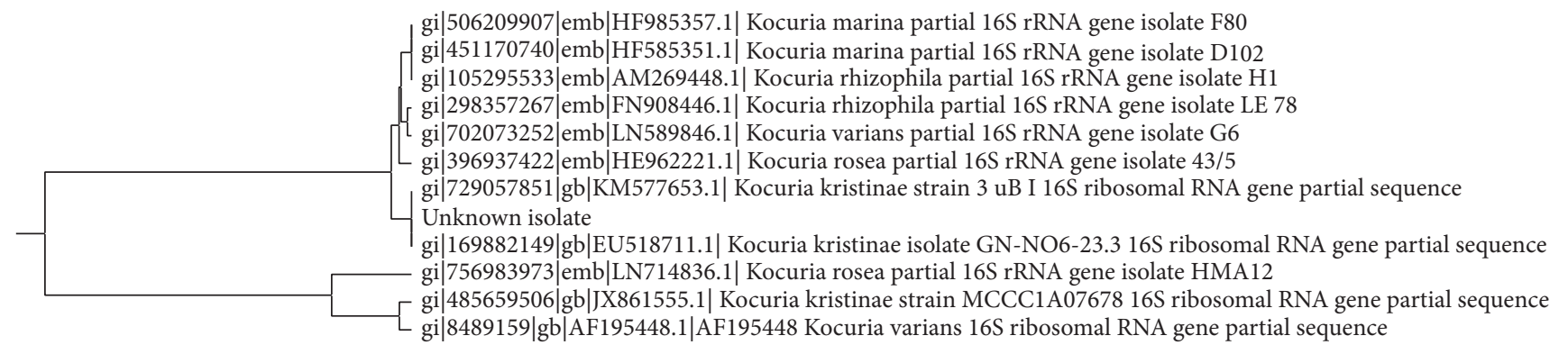

$\begin{array}{lllllll}0.6 & 0.5 & 0.4 & 0.3 & 0.2 & 0.1 & 0.0\end{array}$

FIGURE 1: Evolutionary relationships of taxa. The evolutionary history was inferred using the UPGMA method [10].

Microbiological Methods. Blood culture was withdrawn in the BACTEC Plus aerobic/F and BACTEC Plus anaerobic/F blood culture bottles (Becton, Dickinson and Company, Spain, MD). All bottles were incubated in BACTEC 120 instrument. Blood culture grew Gram-positive cocci on blood and chocolate agar with catalase-positive, coagulasenegative, and typical pigmentation of Kocuria colonies (pale rose) that became more distinct after further $24 \mathrm{hr}$ incubation in $4^{\circ} \mathrm{C}$. Vitek 2 automated identification system (bioMerieux, France) was used to identify the isolate using the Gram positive identification cards (GP cards) and the isolate was identified as Kocuria kristinae (99\%). Susceptibility testing was done using the modified Kirby-Bauer disc diffusion method; the organism was found to be sensitive to cefoxitin, gentamicin, amikacin, ciprofloxacin, levofloxacin, and linezolid but resistant to vancomycin, teicoplanin, rifampicin, amoxicillin/clavulanate, and clindamycin. Phenotypic identification was confirmed by performing a molecular assay, namely, 16S rRNA gene sequencing, as previously described using the primer sets 536f $5^{\prime}$ CAGCAGCCGCGGTAATAC and RP2 5'eACGGCACCTTGTTACGACTT (AccuOligo, Bioneer, Daejeon, Korea), BigDye ${ }^{\circledR}$ Terminator v3.1 cycle sequencing kit (Applied Biosystems, Foster City, CA, USA), and the BigDye Xterminator ${ }^{\mathrm{TM}}$ purification kit (Applied Biosystems, Foster City, CA, USA), and then run on Applied Biosystems 3500 Genetic Analyzer (Applied Biosystems, Foster City, CA, USA). Sequences were analyzed with AutoAssembler software (KB_3500_POP7_BDTv3.mob) and compared using the basic local alignment search tool. Also, a neighborjoining phylogenetic tree with the $16 \mathrm{~S}$ rRNA gene sequences of all Kocuria species using MEGA6 program (Figure 1) was constructed [9-12].

\section{Discussion}

By reviewing the literature, 20 reports on Kocuria infections in humans were found, most of which were in immunocompromised hosts with few reports in otherwise healthy people. The five opportunistic Kocuria spp. are K. kristinae, K. rhizophila, K. rosea, K. varians, and K. marina [3, 13-16].

K. kristinae was first described in 1974 (previously known as Micrococcus kristinae). The bacterium is facultative anaerobic, nonmotile, catalase-positive, and coagulase-negative and is known to cause catheter-related bacteremia and infective endocarditis $[2,17]$.

In the present case report, we describe a case of catheterrelated blood stream infection caused by K. kristinae, complicated by DVT, which was the case in other reported infections caused by Kocuria spp. As the patient had a concomitant urinary tract infection (caused by E. coli), the presented fever could be caused either by one of the organisms or even by the DVT. The patient was not known to be immunocompromised except for antipsychotic drugs which with prolonged use can cause impaired liver functions with mild state of immune system disturbance.

Infection with this organism takes place mostly in immunocompromised patients like K. kristinae bacteremia that occurred in a patient suffering from ovarian cancer. This patient had multiple febrile episodes through a period of six months, all of which grew the organism from several blood cultures and central venous lines (CVLs) [18].

K. kristinae was also described as the cause of acute peritonitis in a patient with end-stage renal failure that had CAPD for two years. The source of contamination was suspected to be touch of the catheter that led to access of bacteria into the peritoneal cavity $[4,19]$.

An immunocompetent pregnant female developed severe K. kristinae intravascular infections with suppurative thrombosis that led to septic pulmonary emboli. These complications followed catheter-related blood stream infection [3].

A recent report of $K$. kristinae bacteremia discussed an infant with a history of prolonged diarrhea complicated with black hairy tongue symptoms [20].

A different access route of $K$. kristinae infection was recently documented in an elderly diabetic patient who developed endocarditis after amputation of a forefoot ulcer and the central venous catheter was not involved [21].

Many recent studies, including ours, correctly identified Kocuria spp. using the Vitek-2 ID-GPC Gram-positive identification card, perhaps due to the recently introduced larger database that allows the identification of additional taxa [22].

Misidentification among members of the Kocuria genus cannot be ruled out as other studies have reported such situations $[5,13,14]$.

As a consequence to the absence of evidence-based guidelines for managing Kocuria infections, cases are managed 
depending on previous experience or similar cases in the literature. These reports suggested the removal of offending catheter and the use of an antibiotic either alone or in combination. Only one report by Szczerba proposed amoxicillin/ clavulanate along with drugs like ceftriaxone, cefuroxime, doxycycline, and amikacin as a first-line therapy against micrococcal infections [23].

Also, there are no specific criteria for interpreting sensitivity assays with Kocuria isolates and only a few investigated the resistance mechanisms expressed in this genus as the one report that postulated decreased cell wall permeability and multidrug efflux pump expressed in these organisms [2]. Another study identified proteins that may be involved in efflux mechanisms [24].

To summarize, Kocuria kristinae bacteremia should be considered especially in liable patients and on repeated isolation. Introduction of newer diagnostic techniques to the microbiology lab leads to better identification of rare pathogens and underestimated ones. Proper diagnosis is the key to better treatment strategies.

\section{Consent}

Informed consent was obtained from the participant included in the study.

\section{Competing Interests}

The authors declare no competing interests.

\section{References}

[1] E. Stackebrandt, C. Koch, O. Gvozdiak, and P. Schumann, "Taxonomic dissection of the genus Micrococcus: Kocuria gen. nov., Nesterenkonia gen. nov., Kytococcus gen. nov., Dermacoccus gen. nov., and Micrococcus cohn 1872 gen. emend," International Journal of Systematic Bacteriology, vol. 45, no. 4, pp. 682-692, 1995.

[2] V. Savini, C. Catavitello, G. Masciarelli et al., "Drug sensitivity and clinical impact of members of the genus Kocuria," Journal of Medical Microbiology, vol. 59, no. 12, pp. 1395-1402, 2010.

[3] R. Dunn, S. Bares, and M. Z. David, "Central venous catheterrelated bacteremia caused by Kocuria kristinae: case report and review of the literature," Annals of Clinical Microbiology and Antimicrobials, vol. 10, article 31, 2011.

[4] A. Carlini, R. Mattei, I. Lucarotti, A. Bartelloni, and A. Rosati, "Kocuria kristinae: an unusual cause of acute peritoneal dialysisrelated infection," Peritoneal Dialysis International, vol. 31, no. 1, pp. 105-107, 2011.

[5] E. S. K. Ma, C. L. P. Wong, K. T. W. Lai, E. C. H. Chan, W. C. Yam, and A. C. W. Chan, "Kocuria kristinae infection associated with acute cholecystitis," BMC Infectious Diseases, vol. 5, article 60, 2005.

[6] R. Tewari, M. Dudeja, A. K. Das, and S. Nandy, "Kocuria kristinae in catheter associated urinary tract infection: a case report," Journal of Clinical and Diagnostic Research, vol. 7, no. 8, pp. 1692-1693, 2013.

[7] R. Ben-Ami, S. Navon-Venezia, D. Schwartz, Y. Schlezinger, Y. Mekuzas, and Y. Carmeli, "Erroneous reporting of coagulasenegative staphylococci as Kocuria spp. by the Vitek 2 system,"
Journal of Clinical Microbiology, vol. 43, no. 3, pp. 1448-1450, 2005.

[8] W. E. Kloos, T. G. Tornabene, and K. H. Schleifer, "Isolation and characterization of micrococci from human skin, including two new species: Micrococcus lylae and Micrococcus kristinae," International Journal of Systematic Bacteriology, vol. 24, no. 1, pp. 79-101, 1974.

[9] R. M. Hassan, M. G. El Enany, and H. H. Rizk, "Evaluation of broad-range 16S rRNA PCR for the diagnosis of bloodstream infections: two years of experience," Journal of Infection in Developing Countries, vol. 8, no. 10, pp. 1252-1258, 2014.

[10] P. H. A. Sneath and R. R. Sokal, Numerical Taxonomy, Freeman, San Francisco, Calif, USA, 1973.

[11] K. Tamura, M. Nei, and S. Kumar, "Prospects for inferring very large phylogenies by using the neighbor-joining method," Proceedings of the National Academy of Sciences of the United States of America, vol. 101, no. 30, pp. 11030-11035, 2004.

[12] K. Tamura, G. Stecher, D. Peterson, A. Filipski, and S. Kumar, "MEGA6: molecular evolutionary genetics analysis version 6.0," Molecular Biology and Evolution, vol. 30, no. 12, pp. 2725-2729, 2013.

[13] K. Becker, F. Rutsch, A. Uekötter et al., "Kocuria rhizophila adds to the emerging spectrum of micrococcal species involved in human infections," Journal of Clinical Microbiology, vol. 46, no. 10, pp. 3537-3539, 2008.

[14] F. Altuntas, O. Yildiz, B. Eser, K. Gündogan, B. Sumerkan, and M. Çetin, "Catheter-related bacteremia due to Kocuria rosea in a patient undergoing peripheral blood stem cell transplantation," BMC Infectious Diseases, vol. 4, article 62, 2004.

[15] C.-Y. Tsai, S.-H. Su, Y.-H. Cheng, Y.-L. Chou, T.-H. Tsai, and A.-S. Lieu, "Kocuria varians infection associated with brain abscess: a case report," BMC Infectious Diseases, vol. 10, article $102,2010$.

[16] Y. L. Ja, H. K. Si, S. J. Haeng et al., “Two cases of peritonitis caused by Kocuria marina in patients undergoing continuous ambulatory peritoneal dialysis," Journal of Clinical Microbiology, vol. 47, no. 10, pp. 3376-3378, 2009.

[17] C. C. Lai, J. Y. Wang, S. H. Lin et al., "Catheter-related bacteraemia and infective endocarditis caused by Kocuria species," Clinical Microbiology and Infection, vol. 17, no. 2, pp. 190-192, 2011.

[18] G. Basaglia, E. Carretto, D. Barbarini et al., "Catheter-related bacteremia due to Kocuria kristinae in a patient with ovarian cancer," Journal of Clinical Microbiology, vol. 40, no. 1, pp. 311$313,2002$.

[19] C. Y. Cheung, N. H. Y. Cheng, K. F. Chau, and C. S. Li, "An unusual organism for CAPD-related peritonitis: Kocuria kristinae," Peritoneal Dialysis International, vol. 31, no. 1, pp. 107-108, 2011.

[20] E. K. Oncel, M. S. Boyraz, and A. Kara, "Black tongue associated with Kocuria (Micrococcus) kristinae bacteremia in a 4-monthold infant," European Journal of Pediatrics, vol. 171, no. 3, p. 593, 2012.

[21] R. Citro, C. Prota, L. Greco et al., "Kocuria kristinae endocarditis related to diabetic foot infection," Journal of Medical Microbiology, vol. 62, no. 6, pp. 932-934, 2013.

[22] M. Boudevrijns, J. Vandeven, J. Verhaegen, R. Ben-Ami, and Y. Carmeli, "Vitek 2 automated identification system and Kocuria kristinae," Journal of Clinical Microbiology, vol. 43, no. 11, p. 5832, 2005 . 
[23] I. Szczerba, "Susceptibility to antibiotics of bacteria from genera Micrococcus, Kocuria, Nesterenkonia, Kytococcus and Dermacoccus," Medycyna Doswiadczalna i Mikrobiologia, vol. 55, no. 1, pp. 75-80, 2003 (Polish).

[24] H. Takarada, M. Sekine, H. Kosugi et al., "Complete genome sequence of the soil actinomycete Kocuria rhizophila," Journal of Bacteriology, vol. 190, no. 12, pp. 4139-4146, 2008. 


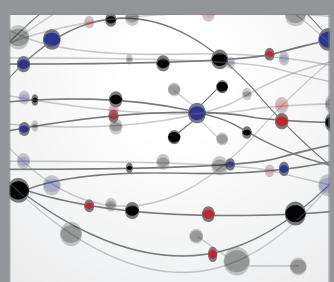

The Scientific World Journal
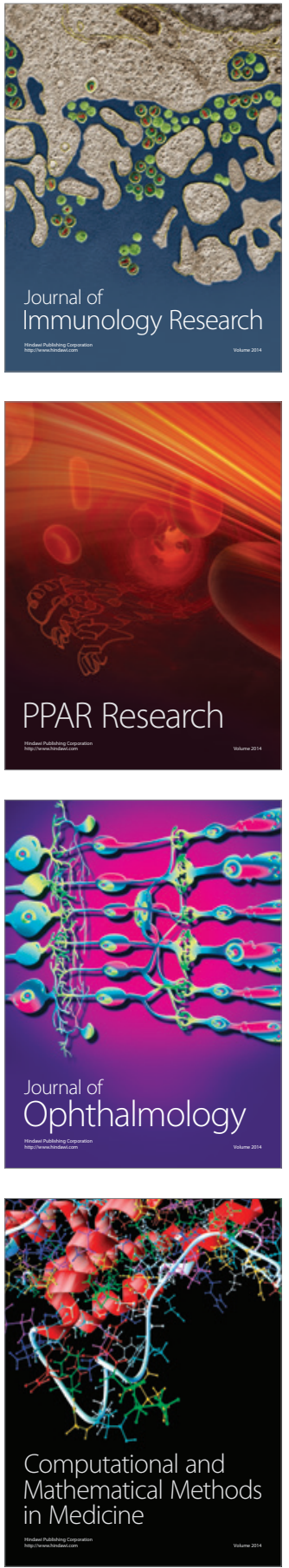

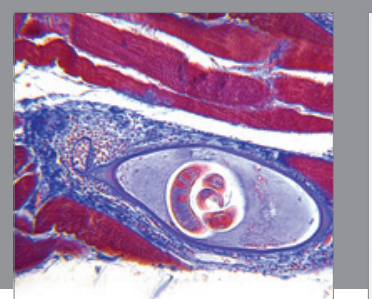

Gastroenterology Research and Practice

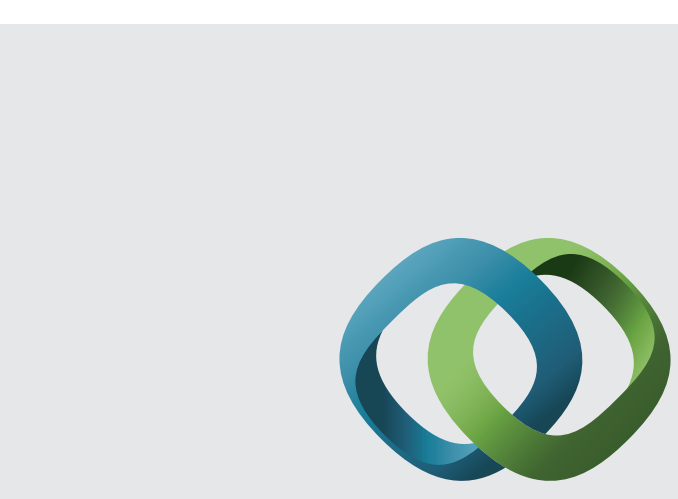

\section{Hindawi}

Submit your manuscripts at

http://www.hindawi.com
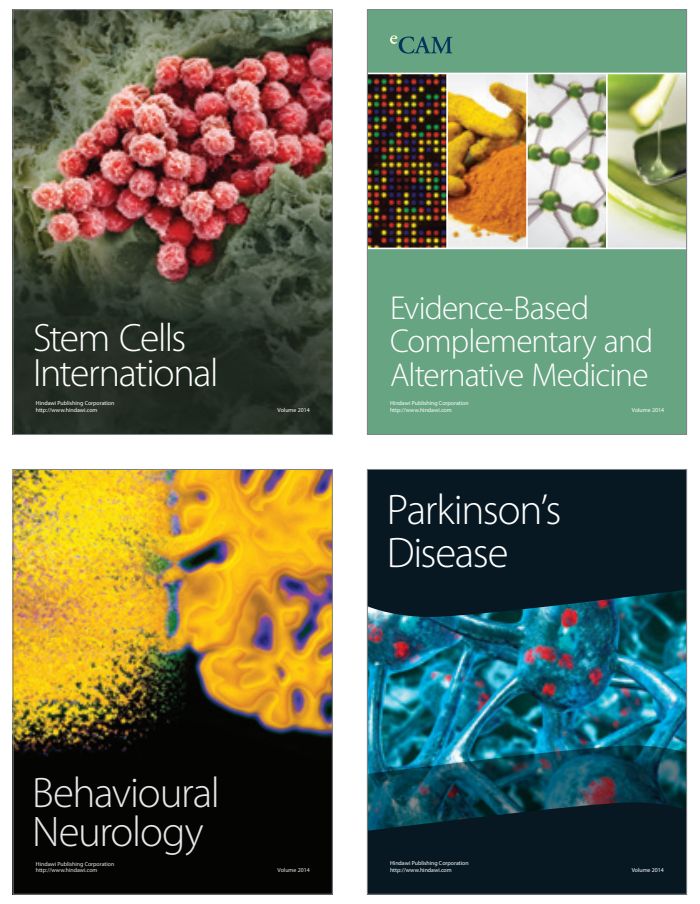
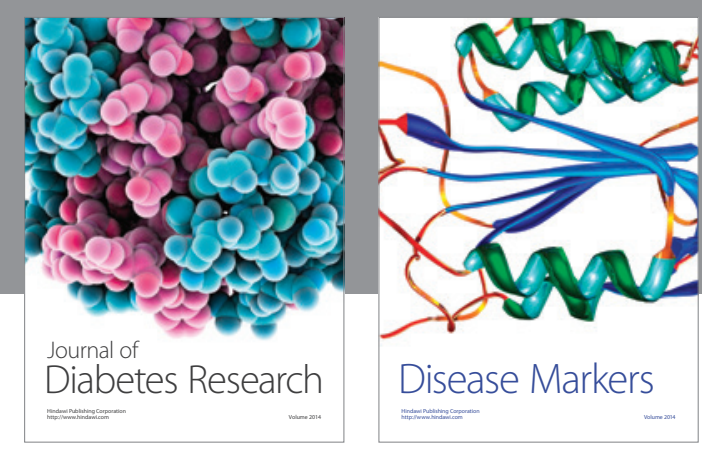

Disease Markers
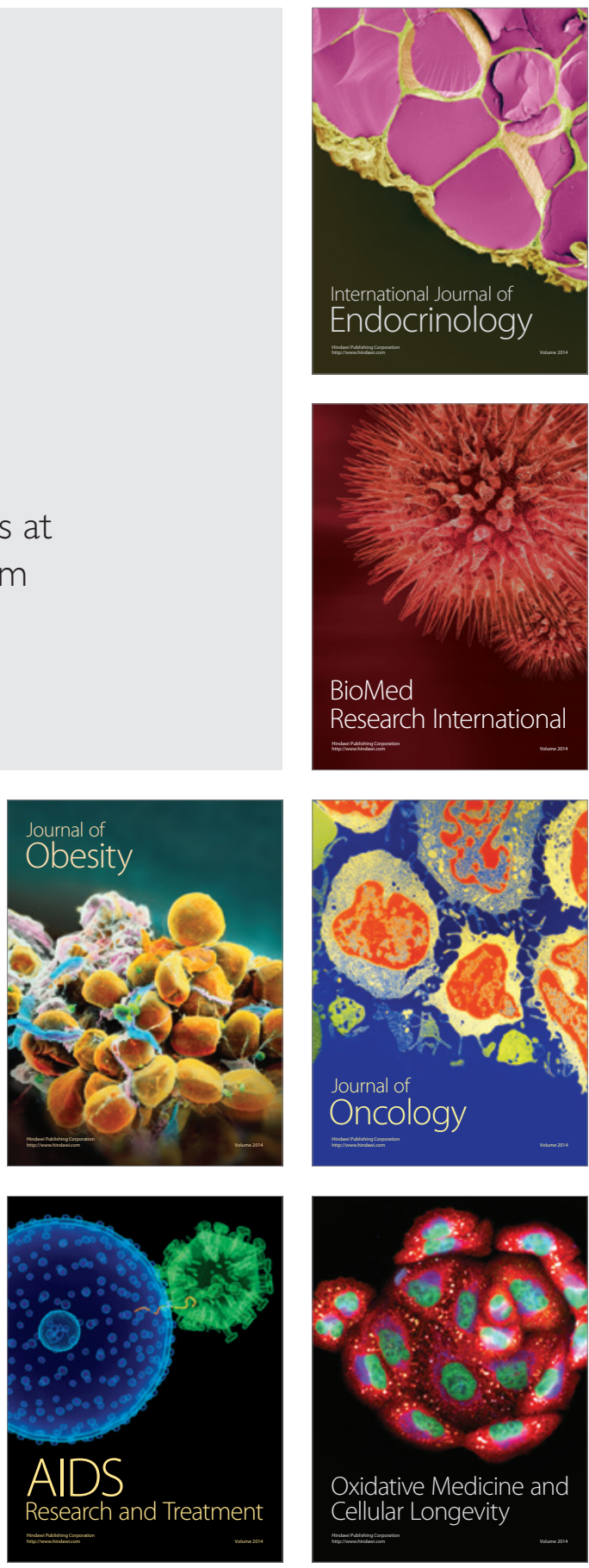